\title{
Spectral K-edge subtraction imaging of experimental non-radioactive barium uptake in bone
}

\author{
Arash Panahifar $^{\mathrm{a}, *}$, Nazanin Samadi $^{\mathrm{b}}$, Treena M. Swanston ${ }^{\mathrm{a}}$, L. Dean Chapman ${ }^{\mathrm{a}, \mathrm{c}}$, David M.L. Cooper ${ }^{\mathrm{a}, *}$ \\ a Department of Anatomy and Cell Biology, College of Medicine, University of Saskatchewan, Saskatoon, Saskatchewan, Canada \\ ${ }^{\mathrm{b}}$ Department of Physics and Engineering Physics, College of Arts and Science, University of Saskatchewan, Saskatoon, Saskatchewan, Canada

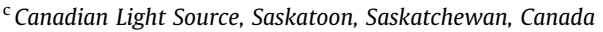

\section{A R T I C L E I N F O}

\section{Article history:}

Received 16 May 2016

Received in Revised form 11 July 2016

Accepted 25 July 2016

Available online 8 August 2016

\section{Keywords:}

K-edge subtraction

Spectral

Bone

Functional imaging

\begin{abstract}
A B S T R A C T
Purpose: To evaluate the feasibility of using non-radioactive barium as a bone tracer for detection with synchrotron spectral K-edge subtraction (SKES) technique.

Methods: Male rats of 1-month old (i.e., developing skeleton) and 8-month old (i.e., skeletally mature) were orally dosed with low dose of barium chloride $\left(33 \mathrm{mg} / \mathrm{kg} / \mathrm{day} \mathrm{Ba}^{2+}\right)$ for 4 weeks. The fore and hind limbs were dissected for imaging in projection and computed tomography modes at $100 \mu \mathrm{m}$ and $52 \mu \mathrm{m}$ pixel sizes. The SKES method utilizes a single bent Laue monochromator to prepare a 550 eV energy spectrum to encompass the K-edge of barium (37.441 keV), for collecting both 'above' and 'below' the K-edge data sets in a single scan.

Results: The SKES has a very good focal size, thus limits the 'crossover' and motion artifacts. In juvenile rats, barium was mostly incorporated in the areas of high bone turnover such as at the growth plate and the trabecular surfaces, but also in the cortical bone as the animals were growing at the time of tracer administration. However, the adults incorporated approximately half the concentration and mainly in the areas where bone remodeling was predominant and occasionally in the periosteal and endosteal layers of the diaphyseal cortical bone.

Conclusions: The presented methodology is simple to implement and provides both structural and functional information, after labeling with barium, on bone micro-architecture and thus has great potential for in vivo imaging of pre-clinical animal models of musculoskeletal diseases to better understand their mechanisms and to evaluate the efficacy of pharmaceuticals.
\end{abstract}

(C) 2016 Associazione Italiana di Fisica Medica. Published by Elsevier Ltd. This is an open access article under the CC BY-NC-ND license (http://creativecommons.org/licenses/by-nc-nd/4.0/).

\section{Introduction}

Molecular imaging is usually associated with nuclear medicine in clinical settings and with the addition of florescence and infrared based imaging in basic sciences research. However, molecular imaging can also be performed using absorption-based X-ray imaging, if a suitable contrast agent (i.e., tracer) is available. Dual energy K-edge subtraction imaging (KES) utilizes the discontinuity in the attenuation coefficient of an element at its K-edge to specifically detect distribution of that element. This can be achieved using synchrotron sources [1-4] and with X-ray tubes [5,6]. The focus of this article is on synchrotron techniques as the more sensitive and developed of the approaches. In KES imaging, two data sets are collected from the sample; one at slightly above and one

\footnotetext{
* Corresponding authors.

E-mail addresses: a.panahifar@usask.ca (A. Panahifar), dml.cooper@usask.ca (D.M.L. Cooper).
}

at slightly below the K-edge of the element of interest, followed by their subtraction. Because the energy difference of these two data sets is typically on the order of several hundreds of $\mathrm{eV}$, the absorption properties of other elements are only negligibly different, therefore, the outcome is the specific map of the tracer. Depending on the nature of experiment, different data collection strategies can be used. Temporal subtraction of the X-ray energies provides more sensitive data, but is only suitable for ex vivo and in vitro experiments due to the need for two separate data acquisitions $[1,2]$. Whereas in simultaneous subtraction methods a narrow range of X-ray energies encompasses the K-edge, thus both data sets are collected at the same time, making this approach more suitable for in vivo experiments [3,7]. Typically, the two Xray energies are prepared by using a focusing bent Laue crystal (as opposed to double Bragg crystals in the temporal method) and placing a splitter in the centre of the beam as they converge towards the sample; the splitter separates the X-ray beams into energies above and below the K-edge. However, this approach 
inherently suffers from a geometrical error due to the interaction of the two beams with the sample at a crossover angle. This is of a less concern if the sample to be imaged is homogenous or composed of materials with similar absorption properties, but becomes problematic at the interfaces of two distinct materials such as bone and soft tissue.

The physiological process of bone turnover (i.e., remodeling) lies at the root of many diseases such as osteoporosis, osteoarthritis, bone cancers, etc. In order to better understand the role of remodeling in bone diseases, we aimed to combine anatomical imaging of bone with functional imaging of new bone formation/turnover at high resolution, after dynamic labeling with non-radioactive barium in rats. Barium, similar to calcium, when bioavailable will incorporate into the hydroxyapatite mineral of newly forming bone, and is normally only present in minute concentrations in the body, thus when administered experimentally it can be used as a tracer of bone formation. To visualize spatial localization of this barium tracer in bone we used a recently modified version of the simultaneous subtraction method called spectral KES (SKES) [4]. This approach utilizes a bent Laue monochromator, but without the splitter, that leads to a faster and more sensitive scans, while also minimizing the 'crossover' artifact. This is possible because of the better energy dispersion of the monochromator which reduces the spectral energy blurring of previous systems. The reduced energy blurring allows the SKES system to use beam closer to the K-edge (less crossover angle) and offers higher flux (no blocked beam due to the splitter). The SKES method has previously been successfully tested on phantoms and ex vivo imaging of lungs of a mouse injected with iodine [4]. In the current study we evaluated simultaneous KES imaging of barium incorporation in skeleton which is more challenging due to the distinctively different attenuation coefficients of bone and soft tissues. This initial proof-of-principle study focused on ex vivo imaging of long bones, with soft tissues attached, to acquire projection and computed tomography (CT) data, but given the promising outcomes, SKES appears as a viable method for imaging bone remodeling in live pre-clinical animal models of musculoskeletal disease.

\section{Materials and methods}

\subsection{Animals}

Barium chloride dihydrate $\left(\mathrm{BaCl}_{2} \cdot 2 \mathrm{H}_{2} \mathrm{O}, \geqslant 99.9 \%\right.$ purity, MW $244.26 \mathrm{~g} / \mathrm{mol}$ ) was purchased from Sigma-Aldrich (Oakville, Ontario). Four groups of healthy Sprague-Dawley male rats were included in the study: 1$)$ Young rats of 1 month-old age $(n=5)$ (i.e., developing skeleton); 2) Adults of 8 month-old age $(n=5)$ (i.e., skeletally mature); 3) Young controls $(n=3)$; and 4) Adult controls $(n=3)$. Barium chloride was dissolved in distilled water and dosed to animals orally with a curved feeding needle at the dosage of $58.5 \mathrm{mg} / \mathrm{kg} /$ day (equivalent to $33 \mathrm{mg} / \mathrm{kg} /$ day free $\mathrm{Ba}^{2+}$ ) for 28 days. Animals were fasted $2 \mathrm{~h}$ before and after the administration to minimize competitive uptake of $\mathrm{Ca}$ by bone during the tracer administration. After euthanization, fore and hind limbs were dissected and frozen for later SKES imaging. The animal use protocol (\# 20110124) was approved by the University of Saskatchewan Animal Research Ethics Board.

\subsection{Spectral K-edge subtraction imaging}

Barium distribution in the bone was evaluated in projection (2D) and CT (3D) mode by SKES technique at the BioMedical Imaging and Therapy (BMIT) bending magnet beamline [8] at the Canadian Light Source (CLS). The schematic set up of the imaging system is presented in Fig. 1. The bent Laue monochromator used in this system provides a narrow spectral range of about $550 \mathrm{eV}$ enabling imaging both 'above' and 'below' energies around the Kedge of barium (37.441 keV) simultaneously (Fig. 2). A $(3,1,1)$ reflection from a $\mathrm{Si}(5,1,1)$ wafer was selected [4]. The size of the beam at focus was about $90 \mu \mathrm{m}$.

Barium phantoms were prepared through serial dilution of barium chloride in distilled water. Although lower concentrations seemed to be detectable, we did not test concentrations lower than $690 \mu \mathrm{g} / \mathrm{mL}$ (Fig. 3). Bone samples were scanned at $100 \mu \mathrm{m}$ pixel size using a flat panel detector. Moreover, projections and CT

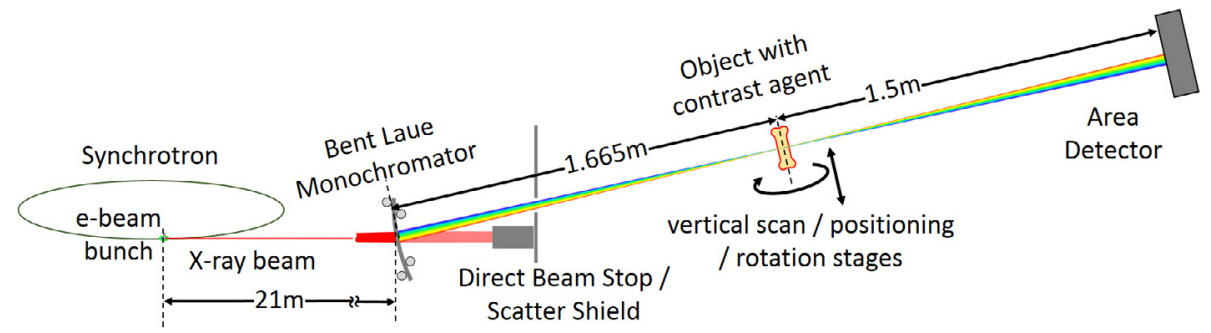

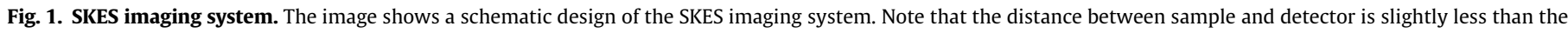

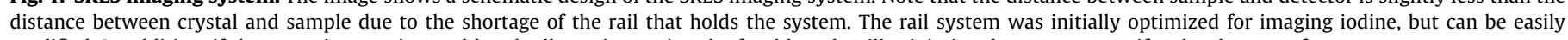
modified. In addition, if the space in experimental hutch allows, increasing the focal length will minimize the crossover artifact by the same factor.

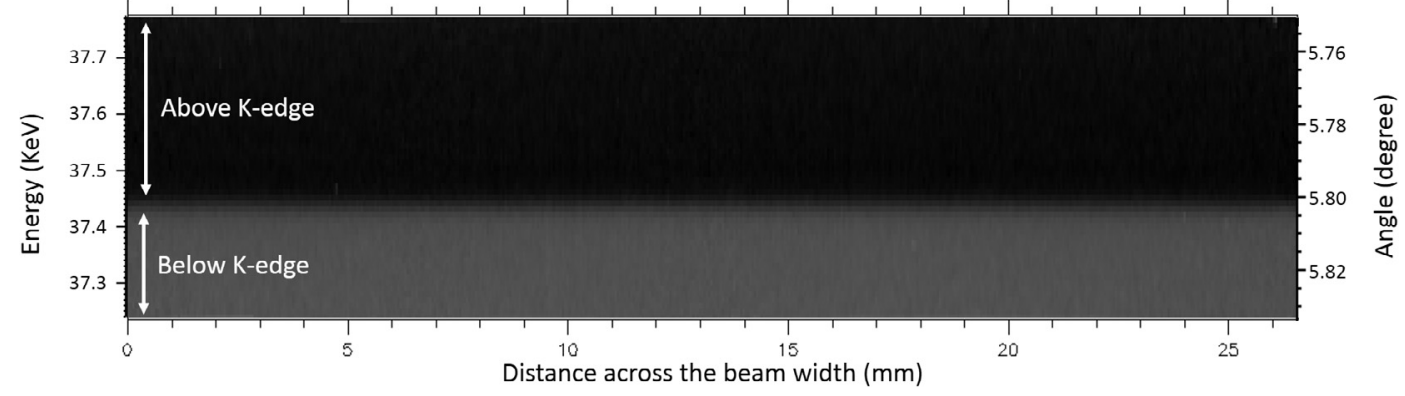

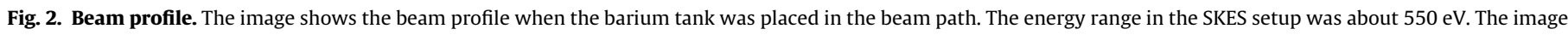
is flat and dark field corrected and was captured with a Hamamatsu ORCA-Flash 4.0 detector (Field of view: $\sim 90 \mu \mathrm{m} * 26 \mathrm{~mm}$, Barium K-edge: 37.44 keV). 


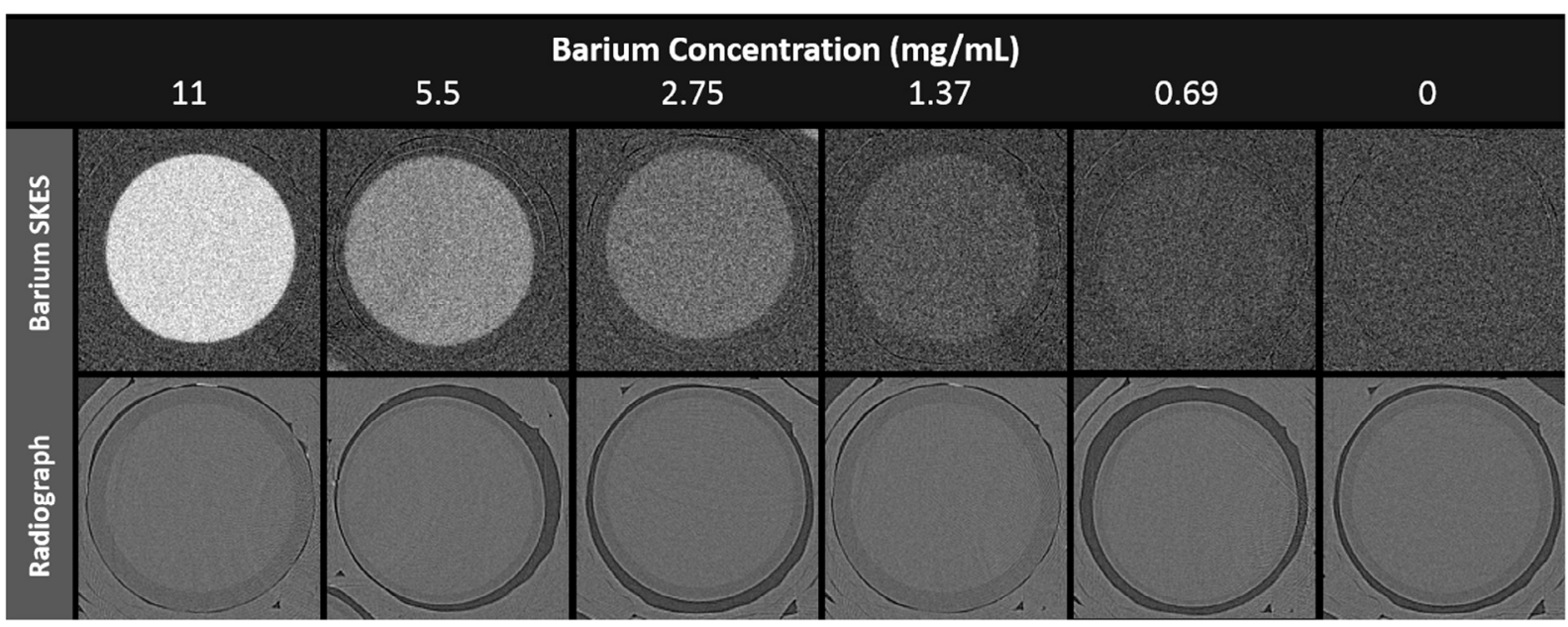

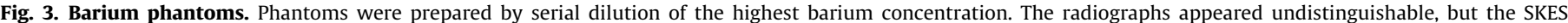

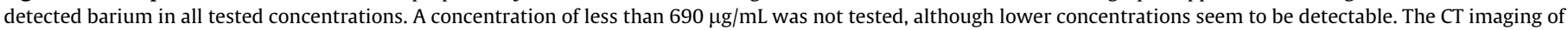
phantoms were performed with the same parameters as imaging bones. Resolution: $52 \mu \mathrm{m}$ voxel size.

images of the same isolated femurs as well as the barium phantoms were acquired by a Hamamatsu ORCA-Flash 4.0 detector at $52 \mu \mathrm{m}$ (binned 4 times from $13 \mu \mathrm{m}$ ) voxel size. Hamamatsu ORCA-Flash 4.0 is a CMOS camera, thus a $10 \mu \mathrm{m}$ thick $\mathrm{Gd}_{2} \mathrm{O}_{2} \mathrm{~S}: \mathrm{Tb}$ scintillator was paired with that. A few frozen animal carcasses were scanned in projection (data not shown) with the flat panel detector as the field of view of the ORCA-Flash camera was not sufficient. During the set up a tank filled with soluble barium was placed in the beam to find the exact barium K-edge energy and then was removed from the beam path. Before each scan 10 projections were collected with no object in the path, as well as 10 projections with no photons, in order to do flat and dark field corrections, respectively. For CT imaging 900 projections over 180 degrees of rotation were collected at constant exposure time of $15 \mathrm{~ms}$. Image processing and reconstruction of CT data were performed in IDL (Exelis Visual Information Solutions, Inc.) using custom written programs and a modified filtered back projection reconstruction method. The image processing methods are reported previously [4].

\section{Results}

The image acquisition time for SKES in projection mode $(15 \mathrm{~cm}$ length) was approximately $30 \mathrm{~s}$ and the total measured radiation surface dose was $0.75 \mathrm{mGy}$. Because the focal spot is small, the sample stage has to move vertically to collect the data, however, the surface dose will remain the same.

Short term exposure to barium at the experimented low concentration led to its incorporation predominantly in newly forming bone such as at the growth plate and trabecular surfaces of articular joints (Fig. 4). In the older animals because the skeleton is fully developed, only remodeling events occur, whereas in the juvenile animals both remodeling and growth (i.e., modeling) events are present. As a result, in the juvenile rats significantly higher concentrations of barium were detected in the bones (Figs. 4 and 5). Moreover, in the adult rats, barium was not detected in the cortical bone, whereas in the juveniles, barium was incorporated both in the cortical and trabecular bone. The exception was in the periosteal and endosteal layers of the cortical bone of adults that also

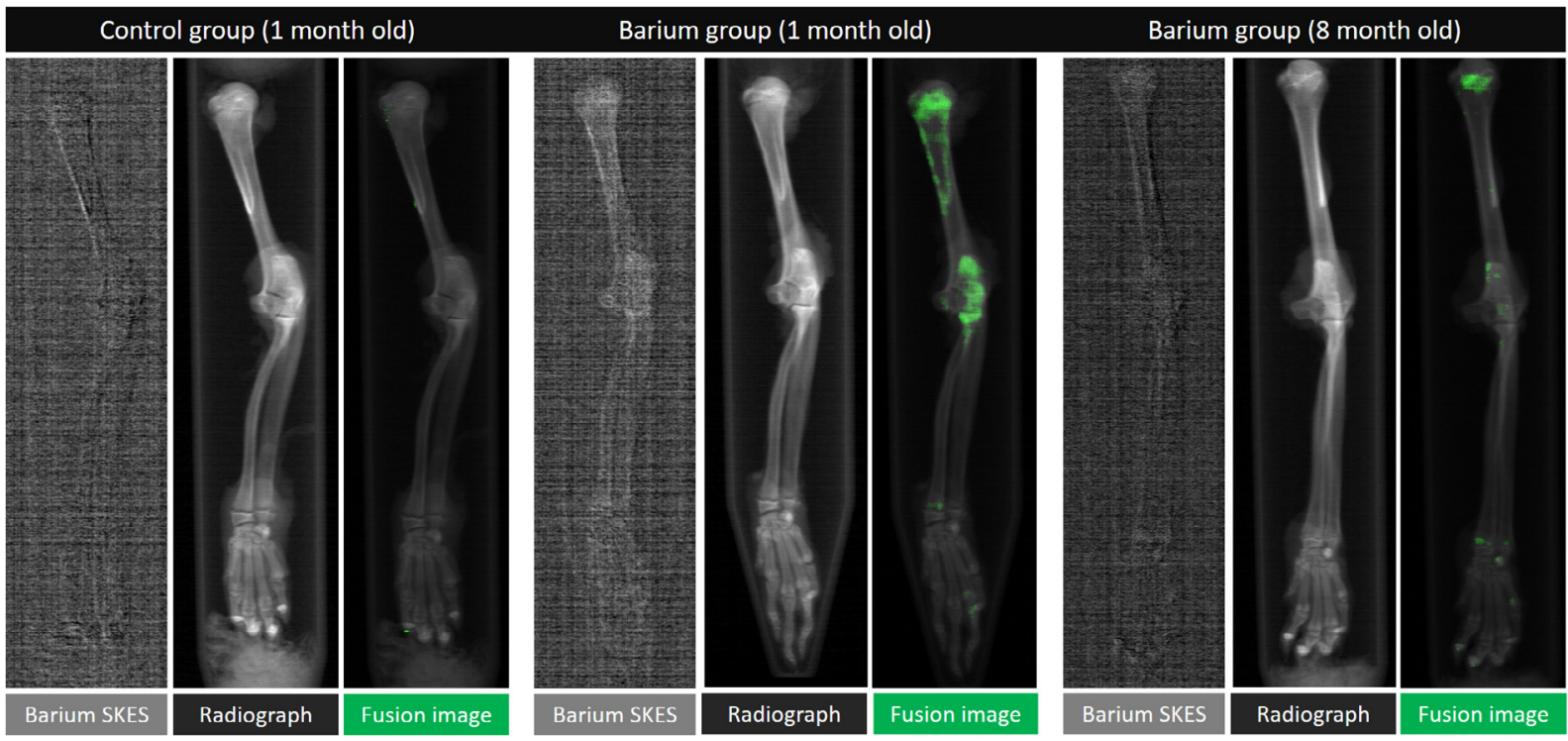

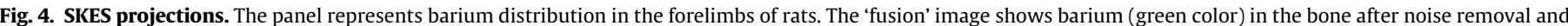

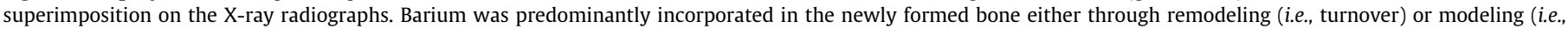
growth) processes. Resolution: $100 \mu \mathrm{m}$. (For interpretation of the references to color in this figure legend, the reader is referred to the web version of this article.) 


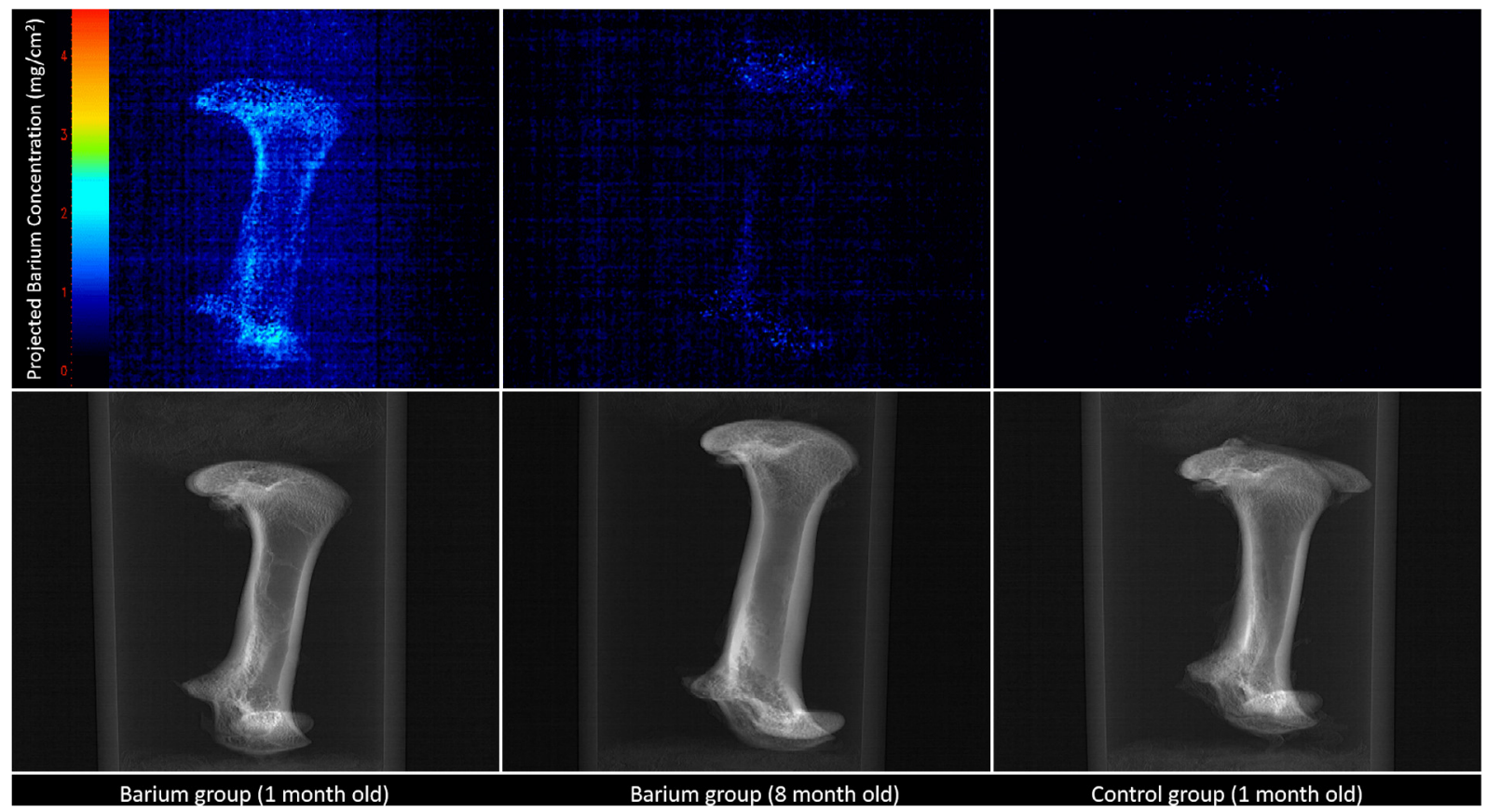

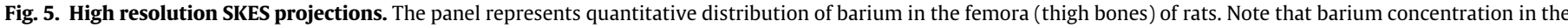

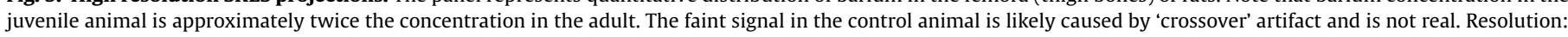
$52 \mu \mathrm{m}$.

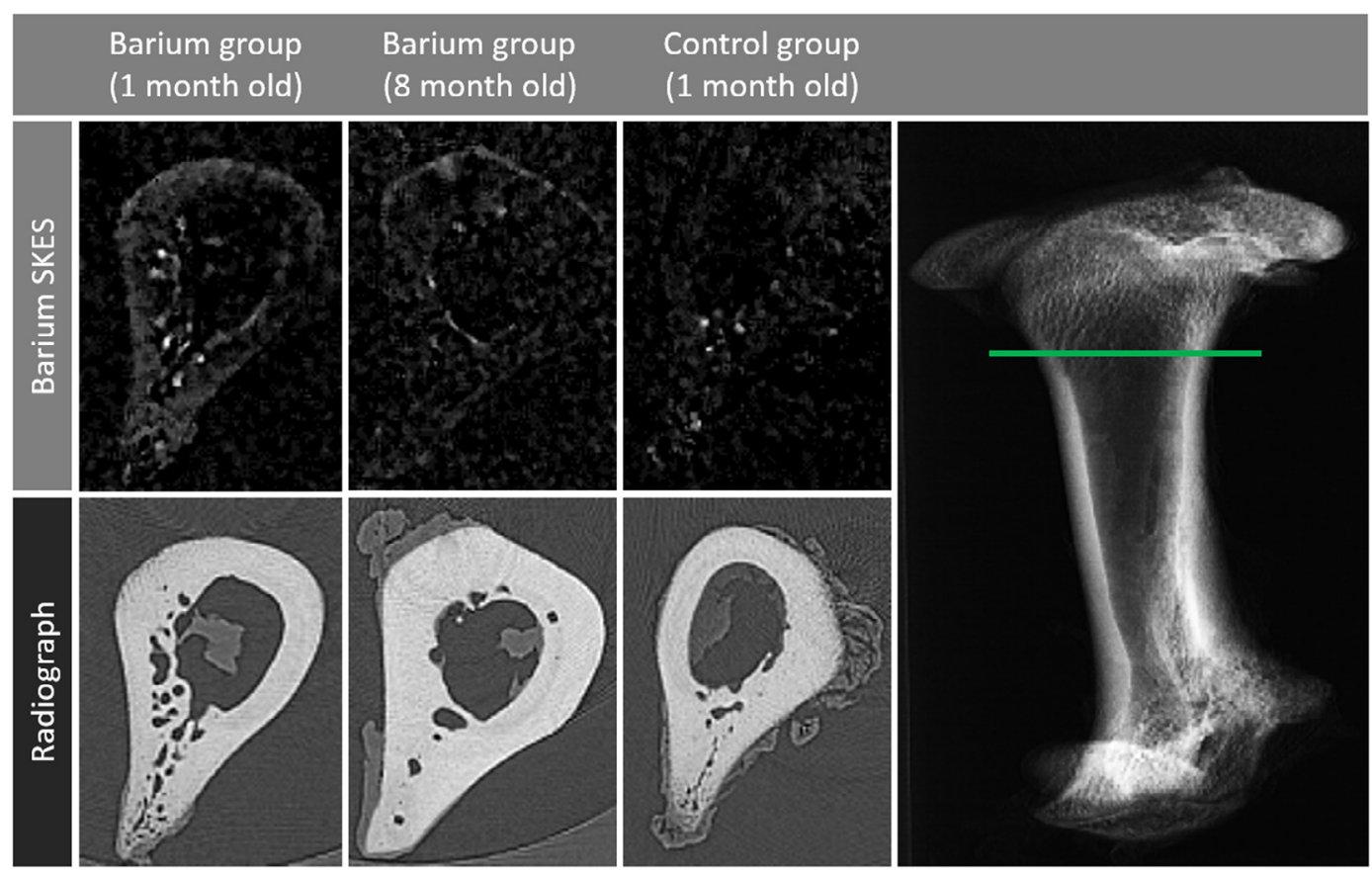

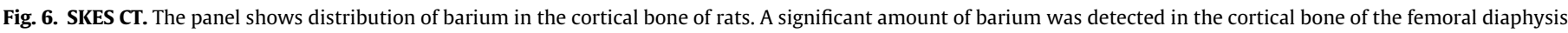

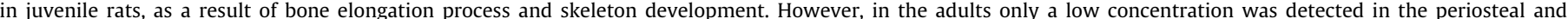

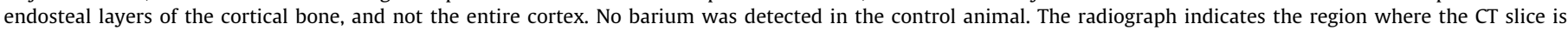
derived from. The barium images are post-processed with a median filter (radius $=1$ ) to suppress the background noise. Resolution: $52 \mu \mathrm{m}$ voxel size.

incorporated barium through the appositional growth process, though at much lower concentration than younger animals (Fig. 6). As expected, no barium was detected in bones of the control groups. Besides the skeleton, barium was also detected in the gastrointestinal tract as its excretion route, and because the animals were euthanized a few hours after the final barium feeding and had not passed the barium.

\section{Discussion}

Barium is typically used in fluoroscopic imaging of gastrointestinal tract in form of insoluble barium sulfate [9]. However, if soluble, barium is taken up by the skeleton and can be viewed as a surrogate for calcium. The attenuation coefficient of barium increases by a factor of 5.2 above its K-edge ( $37.44 \mathrm{keV})$, that along 
with the bone targeting property make barium an interesting experimental tracer to investigate bone turnover. Our group has recently evaluated barium as a bone tracer for detection with temporal KES method [10] and here we utilized a simultaneous subtraction method for that purpose. The soluble form of barium, though toxic above certain quantities, was necessary to use in this study in order to pass it to the bloodstream and ultimately deliver to the skeleton. The main toxic effects of barium are on the cardiovascular system by blocking potassium channels that will cause cardiac arrhythmia and in acute cases death, as well as nephrotoxic effects [11]. Toxicological studies in rats dosed with barium chloride in their drinking water for 13 weeks have found its noeffect-level to be 2000 ppm [11,12]. During that 13-week study water consumption by rats varied, but the average cumulative barium intake for male rats was $2270 \mathrm{mg}$, that accounts for $25 \mathrm{mg} /$ day $\mathrm{Ba}^{2+}$. In our study barium was orally administered to animals to eliminate the variability in water consumption. The dosage was adjusted according to their weights every week. The juvenile and adult rats in this study received the respective average barium doses of $10.6 \mathrm{mg} /$ day and $20.7 \mathrm{mg} /$ day $\mathrm{Ba}^{2+}$ over 4 weeks, that is below the toxic levels. In agreement with the literature, in this study we did not witness any signs of toxicity or discomfort in the treated rats. All rats treated with barium continued to gain weight at the same rate as control rats.

The SKES is a powerful and sensitive method for contrast element imaging. This article presents the application of simultaneous K-edge subtraction for detection of contrast agents in bone. Collecting 2D projections of the bones were simple, accurate, quick, and resulted in low radiation dose. The conventional simultaneous subtraction method uses a bent Laue monochromator with a splitter in the middle that blocks one third of the beam to separate two X-ray beams at above and below the K-edge energy $[3,7]$. In the SKES method no splitter is used resulting in a smaller focal point, higher flux, and technical simplicity. With respect to the latter point, the small BMIT-BM beam height of $7 \mathrm{~mm}$ with a roughly Gaussian distribution posses challenges for implementing a splitter which would remove the most intense portion of the beam [4]. The vertical resolution in this system is limited by the current focal size of $90 \mu \mathrm{m}$, though a smaller focal point could be achieved.

From a medical imaging perspective, the main challenge with the simultaneous KES methods is to minimize the 'crossover' artifact that is created due to the use of bent crystals. The crossover artifact occurs because the imaging system focuses the beam vertically at the object location, therefore, the X-ray path that each ray takes is slightly different in the vertical plane. Since the beam has a range of energies, parts of the object that have strong spatial absorption differences will have differential absorption as a function of energy. It is this energy dependent absorption that allows the contrast agent to be imaged in the object. However, because $\mathrm{X}$-rays reach the sample at a slightly different location it can also causes crossover artifact. If the high energy part of the beam is preferentially absorbed, there will be a true appearance or artifact of the contrast material. Conversely, a preferential absorption of the low energy component of the beam will result in a 'negative' artifact of the contrast material in the image. This is less pronounced in imaging soft tissues such as detection of xenon in lungs $[13,14]$, or heart angiography with iodine $[3,7]$, but becomes problematic at the interface of two tissues with very distinct attenuation coefficients such as bone and soft tissue. Furthermore, in larger objects more parts of the sample are placed out-of-focus, thus resulting in more artifact. In our system, the range of angles at the crossover was minimized by reducing the vertical beam size to be just bracketing the contrast element K-edge value. Also, having good energy dispersive properties offered a well-defined (in angle) width of the K-edge transition which is a few electron- volts in width and quite close to the intrinsic value. Finally, the crossover artifact can be further reduced by focusing the beam at a greater distance to narrow the gap between the 'above' and 'below' X-ray beams. The present system has a relatively short focal length $(\sim 1.5 \mathrm{~m})$ to allow the entire imaging system to be placed within the experimental hutch on a rail system ( $\sim 3 \mathrm{~m}$ length). Increasing the focal length would decrease the overall crossing angle of the beam by the same factor.

Chemical shifts will be small for higher-Z elements such as barium at the K-edge. The small changes in the electronic environment due to bonding will have little effect on the tightly bound $\mathrm{K}$-shell electron transition energy. In addition, the deflection of the imaging beams due to refraction at these energies is quite small. However, we do see refraction or phase contrast effects with this imaging method, but this requires a detector with sufficient spatial resolution to observe phase contrast. A pixel size of $20 \mu \mathrm{m}$ or smaller would allow phase contrast to be observed on the BMIT-BM beamline, but only in the horizontal direction since there is a large range of angles in the vertical direction. Therefore, refraction in the vertical direction will not affect the analysis. In this study the distance between the crystal and object (i.e., at focus) for each scanned sample was optimized in post-processing to virtually move the focus to find the best focal spot that leads to better suppression of the artifact. If different samples of the same bone type were scanned, depending on the size of the bone this value did not change beyond $1-3 \mathrm{~mm}$ among samples. Overall, we witnessed that the 'crossover' artifact is more severe in CT slices and in areas with high structural variations.

In the proposed methodology here, non-radioactive barium was used as a tracer of bone turnover. Functional imaging of bone using radioactive tracers is routinely available with ${ }^{99 \mathrm{~m}} \mathrm{Tc}$-MDP single photon emission computed tomography (SPECT) and ${ }^{18} \mathrm{~F}$ positron emission tomography (PET) at resolutions of $1-2 \mathrm{~mm}$, however, the SKES method offers better spatial resolution and prevents possible complexities of using radioisotopes. The SKES method combined with barium as a tracer is a powerful method to investigate the role of bone in various musculoskeletal diseases to understand the mechanisms of those diseases. Furthermore, the presented methodology provides both anatomical and functional information of the bone micro-architecture in a single scan. Because, this method is non-invasive the samples could be retrieved for subsequent histological evaluation. Non-radioactive barium in sulfate form has been used as an angiographic contrast agent [15,16] and also for imaging micro-damage in bone [17] using synchrotron imaging techniques. However, to the best of our knowledge this is the first report on synchrotron detection of non-radioactive water-soluble barium as a tracer of bone turnover.

\section{Conclusions}

The temporal KES method, while being highly effective for ex vivo samples, is not ideal for in vivo imaging due to the necessity of holding the animal perfectly still for two successive scans - a requirement which is largely unfeasible. The conventional simultaneous KES method has been used in live subjects, but is susceptible to moderate 'crossover' artifact. The presented SKES method scans the X-ray energies simultaneously, minimizing the motion artifact, is quick and is less susceptible to 'crossover' due to the better energy dispersion allowing a smaller range of angles through the object. The SKES method has great potential, after barium administration, for investigation of the role of bone remodeling in musculoskeletal diseases initiation and progression. For example, it can be used for rapid scout scanning of live animals for detection of bone metastasis in cancer animal models and to evaluate treatment efficacy of potential therapeutics. 


\section{Acknowledgments}

This study was supported by the Sylvia Fedoruk Canadian Center for Nuclear Innovation. DMLC and DC are supported, in part, by the Canada Research Chairs program. AP is a Fellow in the Canadian Institutes of Health Research Training grant in Health Research Using Synchrotron Techniques (CIHR-THRUST). The research described in this paper was performed at the Canadian Light Source, which is supported by the Canada Foundation for Innovation, Natural Sciences and Engineering Research Council of Canada, the University of Saskatchewan, the Government of Saskatchewan, Western Economic Diversification Canada, the National Research Council Canada, and the Canadian Institutes of Health Research.

\section{References}

[1] Cooper DM, Chapman LD, Carter Y, Wu Y, Panahifar A, Britz HM, et al. Three dimensional mapping of strontium in bone by dual energy K-edge subtraction imaging. Phys Med Biol 2012;57(18):5777-86.

[2] Panahifar A, Cooper DM, Doschak MR. 3-D localization of non-radioactive strontium in osteoarthritic bone: Role in the dynamic labeling of bone pathological changes. J Orthop Res 2015;33(11):1655-62.

[3] Suortti P, Thomlinson W, Chapman D, Gmür N, Siddons DP, Schulze C. A single crystal bent Laue monochromator for coronary angiography. Nucl Instrum Methods Phys Res, Sect A 1993;336(1-2):304-9.

[4] Zhu Y, Samadi N, Martinson M, Bassey B, Wei Z, Belev G, et al. Spectral K-edge subtraction imaging. Phys Med Biol 2014;59(10):2485-503.

[5] Schlomka JP, Roessl E, Dorscheid R, Dill S, Martens G, Istel T, et al. Experimental feasibility of multi-energy photon-counting K-edge imaging in pre-clinical computed tomography. Phys Med Biol 2008;53(15):4031-47.
[6] de Vries A, Roessl E, Kneepkens E, Thran A, Brendel B, Martens G, et al Quantitative spectral K-edge imaging in preclinical photon-counting x-ray computed tomography. Invest Radiol 2015;50(4):297-304.

[7] Suortti P, Thomlinson W. A bent Laue crystal monochromator for angiography at the NSLS. Nucl Instrum Methods Phys Res, Sect A 1988:269(3):639-48.

[8] Wysokinski TW, Chapman D, Adams G, Renier M, Suortti P, Thomlinson W. Beamlines of the biomedical imaging and therapy facility at the Canadian light source-Part 1. Nucl Instrum Methods Phys Res, Sect A 2007;582(1):73-6.

[9] Barrs TJ. Overview of radiopaque drugs: 1895-1931. Am J Health Syst Pharm 2006;63(22):2248-55.

[10] Panahifar A, Swanston TM, Jake Pushie M, Belev G, Chapman D, Weber L, et al. Three-dimensional labeling of newly formed bone using synchrotron radiation barium K-edge subtraction imaging. Phys Med Biol 2016;61(13):5077-88.

[11] National Toxicology Program. NTP Toxicology and Carcinogenesis Studies of Barium Chloride Dihydrate (CAS No. 10326-27-9) in F344/N Rats and B6C3F1 Mice (Drinking Water Studies). Natl. Toxicol. Program. Tech. Rep. Ser. 1994; 432:1-285.

[12] Dietz DD, Elwell MR, Davis Jr WE, Meirhenry EF. Subchronic toxicity of barium chloride dihydrate administered to rats and mice in the drinking water. Fundam Appl Toxicol 1992;19(4):527-37.

[13] Bayat S, Porra L, Suhonen H, Suortti P, Sovijarvi AR. Paradoxical conducting airway responses and heterogeneous regional ventilation after histamine inhalation in rabbit studied by synchrotron radiation CT. J. Appl. Physiol. (1985) 2009;106(6):1949-58.

[14] Strengell S, Keyrilainen J, Suortti P, Bayat S, Sovijarvi AR, Porra L. Radiation dose and image quality in K-edge subtraction computed tomography of lung in vivo. J Synchrotron Radiat 2014;21(Pt 6):1305-13.

[15] Lafage-Proust MH, Roche B, Langer M, Cleret D, Vanden Bossche A, Olivier T, et al. Assessment of bone vascularization and its role in bone remodeling. Bonekey Rep 2015;8(4):662.

[16] Khoury BM, Bigelow EM, Smith LM, Schlecht SH, Scheller EL, Andarawis-Puri N, et al. The use of nano-computed tomography to enhance musculoskeletal research. Connect Tissue Res 2015;56(2):106-19.

[17] Leng H, Wang X, Ross RD, Niebur GL, Roeder RK. Micro-computed tomography of fatigue microdamage in cortical bone using a barium sulfate contrast agent. J Mech Behav Biomed Mater 2008;1(1):68-75. 